\title{
Self-organized criticality in solar flares: a cellular automata approach
}

\author{
L. F. Morales $^{1}$ and P. Charbonneau ${ }^{2}$ \\ ${ }^{1}$ Canadian Space Agency, Saint-Hubert, Quebec, Canada \\ ${ }^{2}$ Département de Physique, Université de Montréal, C.P. 6128 Succ. Centre-ville, Montréal, Quebec, H3C-3J7, Canada
}

Received: 8 April 2010 - Revised: 21 June 2010 - Accepted: 25 June 2010 - Published: 22 July 2010

\begin{abstract}
We give an overview of a novel lattice-based avalanche model that reproduces well a number of observed statistical properties of solar flares. The anisotropic lattice is defined as a network of vertically-connected nodes subjected to horizontal random displacements mimicking the kinks introduced by random motions of the photospheric footpoints of magnetic fieldlines forming a coronal loop. We focus here on asymmetrical driving displacements, which under our geometrical interpretation of the lattice correspond to a net direction of twist of the magnetic fieldlines about the loop axis. We show that a net vertical electrical current density does build up in our lattice, as one would expect from systematic twisting of a loop-like magnetic structure, and that the presence of this net current has a profound impact on avalanche dynamics. The presence of an additional energy reservoir tends to increase the mean energy released by avalanches, and yield a probability distribution of released energy in better agreement with observational inferences than in its absence. Symmetrical driving displacements are in better conceptual agreement with a random shuffling of photospheric footpoint, and yield a power-law distribution of energy release with exponent larger than 2, as required in Parker's nanoflare model of coronal heating. On the other hand, moderate asymmetrical driving generate energy distribution exponents that are similar to those obtained from SOHO EUV observations.
\end{abstract}

\section{The solar corona: a magnetized environment}

The sun is a magnetic star. Although its magnetic field has little influence on the sun's internal structure, it becomes a dominant dynamical player from the photosphere up to the outer corona. The solar magnetic field is also the energy

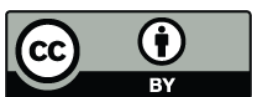

Correspondence to: L. F. Morales

(laura@astro.umontreal.ca) source and engine of all geoeffective eruptive phenomena that collectively make up solar activity. In addition, it modulates the sun's radiative output, especially in the short wavelength range of the electromagnetic spectrum.

In the solar photosphere the energy density is dominated by the plasma, except in regions of strongly concentrated magnetic fields such as sunspots, faculae, and some small magnetic flux concentrations. Rising to the lower corona, these roles are reversed, and the plasma becomes channeled and confined by the coronal magnetic field. This is amply demonstrated by observations of the sun in ultraviolet and soft X-Rays (for example in Lopez Fuentes et al., 2006; De Moortel et al., 2003), which show the corona to be an assemblage of magnetic loops and arcades of various sizes and shapes, anchored in the photosphere and filled with $\sim 10^{6}$ degree Kelvin plasma intermittently heating up to temperatures sometimes reaching $10^{7} \mathrm{~K}$.

Given photospheric temperatures of some $6000 \mathrm{~K}$, and in view of significant coronal conductive and radiative energy losses, an efficient heating mechanism is needed to sustain these high coronal temperatures. The physical nature of this mechanism has remained a puzzle for now over half a century (see Aschwanden, 2006; Klimchuk, 2006; Mandrini et al., 2000; Gomez, 1990 for examples on the diversity of models proposed). What appears certain is that the mechanical energy of photospheric convective motions gets transferred, somehow, to the coronal magnetic field, where it accumulates gradually until it is released through MHD or plasma instabilities to heat the coronal gas. Because the latter is a good electrical conductor, the coronal magnetic field has been argued to reach a highly tangled, topologically complex state in response to stochastic motions of its photospheric footpoints. Energy release would then take place in a large number of small electrical current sheets forming spontaneously in regions of magnetic tangential discontinuities. Two papers from E. N. Parker (Parker, 1983, 1988) give a succinct overview of this idea, together with estimates of the energetic and dynamical requirements.

Published by Copernicus Publications on behalf of the European Geosciences Union and the American Geophysical Union. 
The reconnection presumably taking place at such small current sheets alters the physical conditions in the vicinity of the reconnection site, which may push other neighboring current sheets beyond their stability threshold and trigger more reconnection events, in classical avalanching style. The resulting energy release can then be interpreted as a solar flare. This idea, first proposed and explored by Lu and Hamilton (1991) using a sandpile-like cellular automaton (CA) driven to a state of self-organized criticality (SOC), naturally yields a scale-free distribution of released energy. This provides a natural explanation for the observationally-inferred powerlaw distribution of flare energy, which spans some eight orders of magnitude (see Aschwanden et al., 2000).

In subsequent years many CA for solar flares were proposed. Although different in many details most of them shared the same common characteristics (for reviews on the subject see Charbonneau et al., 2001; Podladchikova and Lefebre, 2006). Although such models can reproduce certain aspects of observed solar flares statistics, their simplicity of design led to ambiguous or problematic interpretative scenarios when attempting to link the model back to magnetohydrodynamics. For example, if one associates the nodal variable in the $\mathrm{Lu}$ and Hamilton (1991) CA with the magnetic field, then the condition $\nabla \times \boldsymbol{B}=0$ ends up violated; if the nodal variable is instead identified with the magnetic vector potential, then ambiguities appear in the calculation of magnetic energy release. Such physical considerations motivated us to design a novel SOC model for solar flares (Morales and Charbonneau, 2008), in which the dynamical elements are linear strands of interconnected nodes representing magnetic fieldlines, rather than isotropic nodal sampling of magnetic field intensity. Forcing takes place by introducing horizontal displacements ( $\delta_{-}$to the left or $\delta_{+}$to the right) at randomly selected nodes. We could show that this model produces spatially and temporally intermittent, avalanche-like release of magnetic energy with frequency distributions of avalanche size parameters in the form of power laws with indices comparing to observationally inferred values. Moreover, and unlike in most of previous models, avalanches liberated a significant fraction of the total energy stored in the lattice.

One of the most natural geometrical interpretation of our 2-D anisotropic lattice is that it represents the cartesian mapping of the outer magnetic flux surface of a straightened coronal loop, with the vertical direction on the lattice corresponding to the direction along the loop axis, and the horizontal to the azimuthal direction along the perimeter of the loop crosssection. Reconstructions of synthetic flare images under this geometrical Ansatz have been found to compare favorably to observations, in the sense that the size distribution and fractal indices of the flaring areas matched reasonably well with values inferred from UV and EUV imaging of flaring coronal loops (Morales and Charbonneau, 2009). Any asymmetry in forcing displacements in the horizontal direction would then amount to a systematic sense of twisting of the magnetic fieldlines around the loop axis. Such a systematic, large-scale twist should then result in buildup of large-scale net electrical current density flowing along the loop axis. What is then the impact of such a twist on the avalanching dynamics? This is the primary question addressed in this paper.

We begin (Sect. 2) with an overview of the several elements of the CA, and associated avalanching dynamics, focusing in particular on the avalanching characteristics that can be compared to solar flare observations. In Sect. 3 we systematically explore the effect of asymmetric forcing on the characteristics of the statistical properties of avalanches, and offer a well-defined physical interpretation for the results obtained, grounded in magnetohydrodynamics. In Sect. 4 we offer a speculative but physically plausible scenario linking our model results to observed patterns of energy release in the solar corona.

\section{The new cellular automaton}

We begin this section by introducing the new SOC model for solar flares whose main statistical properties we studied in Morales and Charbonneau (2008). The model consists in a 2-D lattice of size $N \times N$ that (initially) forms a network of equally spaced vertically interconnected nodes representing a magnetic flux strand. Periodic boundary conditions are applied in the horizontal direction. The driving process is performed by introducing horizontal displacements at randomly selected nodes. The associated lengthening of the flux strands amounts to injecting energy into the lattice. Eventually, crossings between two or more flux strands at certain lattice sites will occur. The angle $\Theta$ subtended by such crossing strands is a measure of the local intensity of electrical current density $\left(\boldsymbol{J}=\frac{1}{\mu_{0}} \nabla \times \boldsymbol{B}\right)$, so that the magnitude of $\Theta$ is used as a stability criterion (Parker, 1988; Dahlburg et al., 2005); whenever $\Theta$ exceeds some preset value $\Theta_{c}$, we mimic magnetic reconnection through a cut-and-splice operation on the two flux strands, and displace one of the two nodes away from the unstable site, so as to lower the magnetic energy and restore local stability. This, in turn, can produce new unstable crossing angles at neighboring lattice sites, which are then themselves cut, spliced and displaced, producing a typical avalanche. Driving is suspended during avalanches, as in classical stop-and-go CA. The implied separation of timescales between driving and forcing is well supported in the solar coronal context, with photospheric fluid motions having typical timescales ranging from tens of minutes to days, and flare onset taking place on timescales of seconds.

Energy release at an unstable site within our pseudocoronal loop really represents the energy released at the reconnection sites themselves, which in the case of real flaring loops are indeed often located in the corona proper, and usually visible in hard X-rays (see Figs. 13.25 and 13.34 in Aschwanden, 2006 for schematics and observations). On the other hand, the peak EUV emission produced by solar 

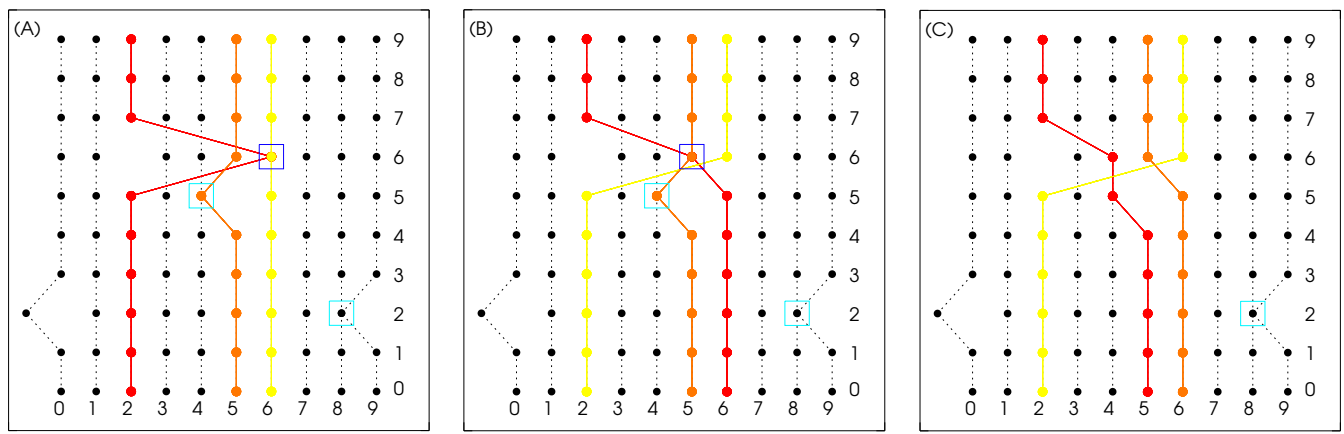

Fig. 1. A two iteration-time lasting avalanche for a small lattice, with driving parameters $\delta_{+}=4$ and $\delta_{-}=-1$. Strands are numbered from left to right and nodes along a given strand from top to bottom. In panel (A) the strand 5 has suffered a perturbation to the left in node $(5,5)$ now located at lattice site $[4,5]$. In panel (A) node $(2,6)$ has been displaced four units to the right. Open squares indicate the nodes where two or more strands meet and form an angle. $\Theta_{[6,6]} \sim 2.65$ is the angle formed by strands 2 and 6 at site $[6,6]$. $\Theta_{[8,2]]}=\frac{\pi}{2}$ is formed between strands 8 and 9 and $\Theta_{[4,5]}=\frac{\pi}{2}$ is formed by strands 4 and 5. Since for this example $\Theta_{C}=2.25$ the only unstable site is [6,6].

In panel $(\mathbf{B})$ site $[6,6]$ is no longer unstable. Strands 2 and 6 have reconnected and the node $(6,6)$ belonging to strand 2 has been displaced to the left. With this displacement a new unstable site appears at $[5,6]$ with $\Theta_{[5,6]} \sim 2.35$ formed by strands 5 and 6 .

In panel $(\mathbf{C})$ the instability has been eliminated: strands 5 and 6 have reconnected and the node $(5,6)$ belonging to strand 5 has moved one unit to the left. At this point there are no more unstable nodes in the lattice and perturbation re-starts.

flares usually originates from lower down, as charged particle accelerated at the reconnection sites are channelled downward and thermalize in the lower corona and chromosphere. Nonetheless, under the assumption that power radiated in the EUV spectral range is proportional to the energization at the reconnection sites, we expect that the statistics of energy release events extracted from our simulations can be legitimately compared to those inferred observationally from EUV and soft X-Ray flare observations (Aschwanden, 2006).

The manner in which displacements are introduced merits further discussion, as it is central to the model results to be discussed below. Once a node has been selected for perturbation, it is displaced either to the right or left, with equal probability, but the size of the left $\left(\delta_{-}\right)$and right $\left(\delta_{+}\right)$ displacements are not necessarily the same. If $\left|\delta_{-}\right|=\left|\delta_{+}\right|$, then nodes execute an unbiased 1-D random walk, so that the mean square displacement should increase linearly in time but the mean displacement remains zero. If on the other hand $\left|\delta_{+}\right|>\left|\delta_{-}\right|$(say), then a net mean displacement builds up, which, under our geometrical interpretation of the lattice, amounts to a net twist about the vertical direction corresponding to the loop's axis.

In Fig. 1 we show an illustrative example of the model in operation, more specifically the triggering and evolution of a small avalanche produced in a $10 \times 10$ lattice. Nodes are labeled using a vectorial index $\boldsymbol{k}=(i, j)$, where the index $i$ labels a magnetic flux strand and the index $j$ the position along the $i$-th strand. Lattice sites are denoted by a pair of discrete $[\mathrm{x}, \mathrm{y}]$ cartesian coordinates. The Figure illustrates how a perturbative displacement, here of node $(2,6)$ relocated to lattice position $[6,6]$ produces an unstable angle between strands 2 and 6 at that lattice site (panel A). Displacement of the node back to site [5, 6] eliminates the unstable crossing angle, but produces a new one, now between strands 2 and 5 (panel B). Further displacement of node $(2,6)$ to the left finally restores stability. Note that the lattice connectivity is now different from what it was in A, and different from what it would had been had node $(2,6)$ simply been displaced two lattice units to the right on panel A.

In what follows we run simulations in order to examine the impact of different perturbation amplitudes over the avalanche properties. In particular, we compare and contrast avalanching behavior and energetics for the symmetric driving case $\delta_{-}=-1$ and $\delta_{+}=1$ to that emerging in the case of asymmetric driving, specifically $\delta_{-}=-1$ and $\delta_{+}=5,4$ and 3 .

\section{Model results}

In Morales and Charbonneau (2008) we have shown that on large enough lattices, statistical properties of avalanches do not depend on the lattice size $(N)$ or the threshold angle $\left(\Theta_{c}\right)$. Accordingly, in this work we perform simulations using a fixed lattice size $N=64$ and fixed angle $\Theta_{c}=2 \mathrm{rad}$. All simulations begin with a configuration of vertically-oriented, straight flux strands parallel to one another. In Fig. 2 we show the time series of lattice energy and energy release for the symmetrical case and for an asymmetrical case $\left(\delta_{+}=3\right.$ and $\left.\delta_{-}=-1\right)$. Although the lattice energy is of the same order of magnitude in both cases, the energy release is dramatically increased when the perturbation amplitude is nonsymmetrical. Moreover, the mean value of both the lattice energy and energy release increases as $\delta_{+}$becomes larger, as illustrated in Fig. 3. While this may have been expected given that a higher displacement $\delta_{+}$implies a greater rate of energy input into the lattice, it is interesting that lattice energy and mean energy release do not simply scale up with $\delta_{+}$. 

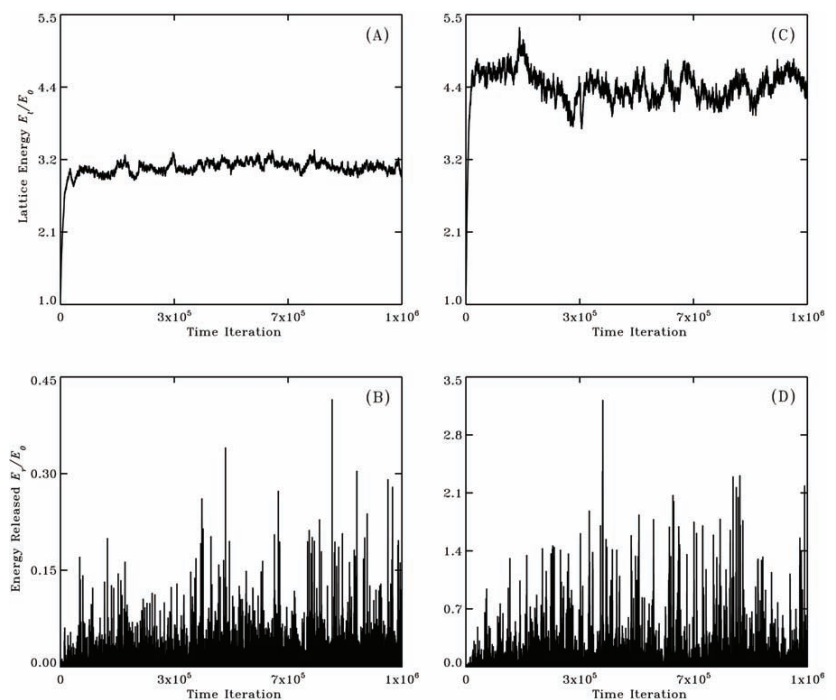

Fig. 2. Time series of total lattice energy and energy released in avalanches in a $64 \times 64$ node lattice with stability threshold angle $\Theta_{\mathrm{c}}=2 \mathrm{rad}$. On the left side amplitudes combination was $\delta_{+}=1$ and $\delta_{-}=-1$, corresponding to symmetrical driving displacements, and on the right $\delta_{+}=3$ and $\delta_{-}=-1$, yielding asymmetrical driving. Note the different vertical scales between panels (B) and (D).
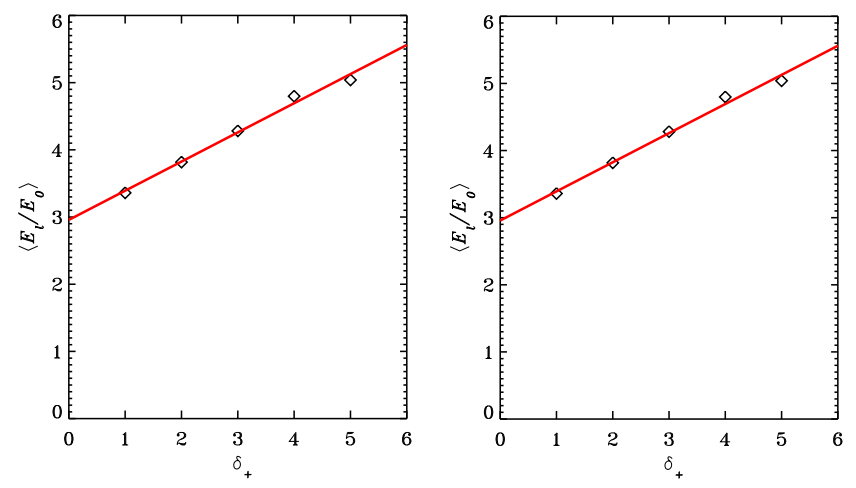

Fig. 3. Mean value of the lattice energy versus right perturbation amplitude $\left(\delta_{+}\right)$. Best fit obtained by performing linear regression analysis are also shown. On the right the mean value of the energy release versus $\delta_{+}$and the quadratic fit performed.

This is shown on Fig. 3, which reveals that the mean value of the lattice energy grows linearly with $\delta_{+}$(slope +0.43 and a correlation coefficient $r=0.995$ ), while the mean value of the energy release grows quadratically, i.e., $<E_{\mathrm{r}} / E_{0}>=a \delta_{+}^{2}$, with quadratic coefficient $a=0.0022 \pm 0.0003$. This indicates that the lattice driven asymmetrically does not simply produce larger avalanches because it accumulates more energy; something else is at play, relating to the avalanching dynamics per se.

We now turn to the global characterization of avalanches. We shall employ the typical quantities used in avalanche analysis: the peak energy release $(P)$ which is the maximum energy released in a single iteration in the course of
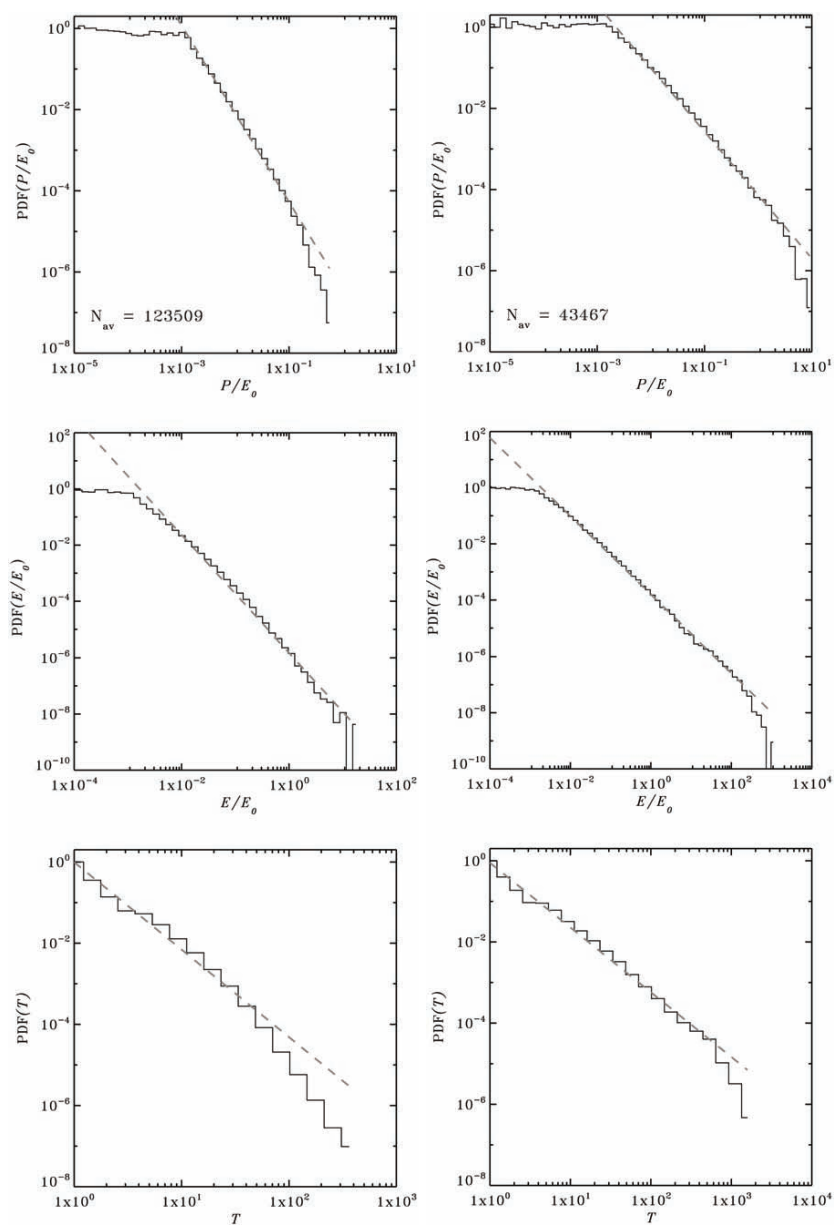

Fig. 4. Probability distribution functions of avalanche size measures, $E, P$ and $T$ for two runs: $\delta_{+}=5$ (right) and $\delta_{+}=1$ (left). Statistics is based on simulations spanning $10^{6}$ iterations.

an avalanche; the total energy $(E)$ which corresponds to the sum of all energy released at every unstable site in the course of an avalanche; and the duration $(T)$ which is the number of iterations from the onset of the avalanche to the recovery of stability across the whole lattice. We extract this quantities for each avalanche in the time series of energy release produced by the model and build the probability distribution functions (hereafter PDF) for $E, P$ and $T$. In Fig. 4 we plot the frequency distributions obtained for two extreme cases: $\delta_{+}=1$ and $\delta_{+}=5$. In that figure it is apparent that the PDFs for $E, P$ and $T$ all take the form of power-law:

$\operatorname{PDF}(X) \propto X^{-\alpha_{X}}, \quad X \in\{E, P, T\}$.

in columns 2 to 4 of Table 1 we list the associated power-law indices obtained for the all the simulations performed.

Examination of Table 1 readily reveals that the values of the $\alpha$ exponents decrease as the driving amplitude $\left(\delta_{+}\right)$increases. Moreover this effect is more dramatic when comparing the symmetrical to any of the asymmetrical examples. This result is produced because of the fact that symmetrical 
Table 1. Power-law indices for total energy $(E)$, peak energy $(P)$ and duration $(T)$ of avalanches. Also mean vertical current density $\left(J_{\mathrm{Z}}\right)$ and its standard deviation $\left(\sigma_{J_{\mathrm{Z}}}\right)$.

\begin{tabular}{lccccc}
\hline $\begin{array}{l}\text { Driving } \\
\delta_{+}\end{array}$ & $\alpha_{E}$ & $\alpha_{P}$ & $\alpha_{T}$ & $\left\langle J_{\mathrm{Z}}\right\rangle$ & $\sigma_{J_{\mathrm{Z}}}$ \\
\hline 1 & $2.09 \pm 0.03$ & $2.17 \pm 0.03$ & $2.16 \pm 0.09$ & $-1.30 \times 10^{-3}$ & 0.20 \\
2 & $1.74 \pm 0.03$ & $1.76 \pm 0.01$ & $1.80 \pm 0.09$ & $-2.56 \times 10^{-2}$ & 0.23 \\
3 & $1.54 \pm 0.02$ & $1.72 \pm 0.02$ & $1.77 \pm 0.07$ & $-7.90 \times 10^{-2}$ & 0.24 \\
4 & $1.47 \pm 0.01$ & $1.67 \pm 0.02$ & $1.73 \pm 0.04$ & $1.25 \times 10^{-1}$ & 0.22 \\
5 & $1.39 \pm 0.01$ & $1.58 \pm 0.02$ & $1.59 \pm 0.03$ & $1.78 \times 10^{-1}$ & 0.23 \\
\hline
\end{tabular}

driving causes more crossings (per unit time) than asymmetrical one, therefore the population of small energetic short lived avalanches grows and, consequently, the PDFs are steeper. When forcing is strongly asymmetrical, all lines tend to stretch in the same direction, and therefore tend to cross less often, but when they do, they liberate more energy since they have been subjected to greater stretching. Moreover, for larger values of $\delta_{+}$the generated avalanches are longerlived. This occurs because for larger values of $\delta_{+}$the process of relaxation to an stable state will involve more cut-splicedisplace steps than with smaller values of $\delta_{+} ;$Fig. 5 offers a simple example demonstrating why this is so.

Let us now return to our geometrical interpretation of the lattice as representing the cartesian mapping of the outer magnetic flux surface of a straightened coronal loop. Assuming that the cylinder's radius $(R=N-1 / 2 \pi)$ is constant and using cylindrical coordinates with origin in the center and bottom of the cylinder; the magnetic field of each line can be expressed as:

$\boldsymbol{B}=B_{\phi}(\phi, z) \hat{\phi}+B_{\mathrm{z}}(\phi, z) \hat{z} ;$

thus the z-component of the current is:

$J_{\mathrm{Z}} \propto \frac{1}{R} B_{\phi}(\phi, z)$.

In a quasi-stationary MHD system Maxwell's displacement current can be neglected, and electrical charge conservation implies $\nabla \times \boldsymbol{J}=0$, so we simply evaluate Eq. (3) at the bottom of the lattice, by projecting horizontally the fieldline segment connecting the first and second node along each strand. We then compute the mean vertical current density $<J_{Z}>$ by summing the $J_{\mathrm{Z}}$ so evaluated horizontally across the lattice:

$$
<J_{\mathrm{Z}}>=\frac{1}{N} \sum_{j=1}^{N} J_{\mathrm{Z}}
$$

We calculate this quantity at every time step in the $\delta_{-}=1$ and $\delta_{+}=5,4,3,2$ simulation, and then perform a temporal average over the portion of the simulations in the statistically stationary state. These temporal means are listed in the rightmost column of Table 1 . The mean vertical current $<J_{\mathrm{Z}}>$ is found to increase significantly with increasing
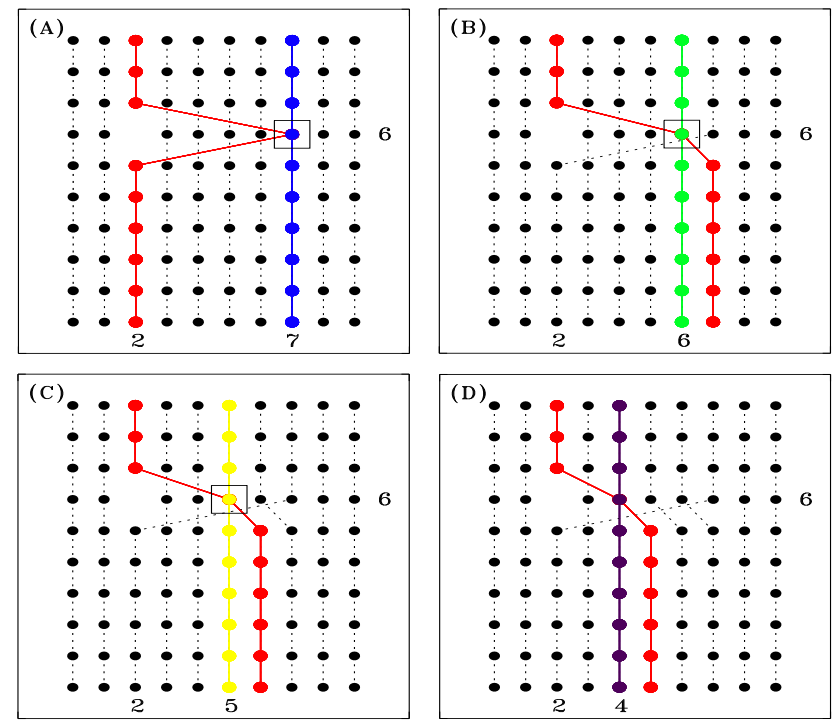

Fig. 5. Simple scheme that explains how a driving of $\delta_{+}=5$ can generate in an otherwise unperturbed strands an avalanche lasting at least three iterations. In panel (A) $\Theta_{[7,6]} \sim 2.75 \mathrm{rad}$ then being $\Theta_{\mathrm{C}}=2$ rad the node $[7,6]$ is unstable so the redistribution rule is applied leading to the configuration shown in panel (B). At that point $[7,6]$ is no longer unstable but $\Theta_{[6,6]} \sim 2.11 \mathrm{rad}$ so redistribution goes on leading to a new unstable node $[5,6]$ with $\Theta_{[5,6]} \sim 2.03 \mathrm{rad}$ forcing another redistribution step. Finally, in panel (D) $\Theta_{[4,6]} \sim 1.892$ so driving resumes.

$\delta_{+}$, going from $\sim-1.3 \times 10^{-3}$ at $\delta_{+}=1$ to $\sim 1.25 \times 10^{-1}$ at $\delta_{+}=4$. This implies that a large-scale vertical electrical current density does build up along the loop axis. Even though the value of the mean current remains of the order of the standard deviation even at $\delta_{+}=5$, the trend is clear, and we expect that better statistics can be obtained by carrying out longer simulation runs.

The presence of this current density has a significant impact on the energetics of avalanches. Examination of the time series of $<J_{Z}>$ reveals that this quantity tends to decrease as the avalanches unfold, suggesting that the large-scale electrical current gets tapped into as an additional energy reservoir to enhance the magnetic energy liberated during avalanches. This is illustrated on Fig. 6, showing the correlation between total liberated energy $E$, and the corresponding differential in mean electrical current in the course of each avalanche. A significant correlation $(r=+0.6)$ is obtained in the case of asymmetrical driving (Fig. 6b), while the correlation is much weaker for symmetrical driving $(r=+0.33$, Fig. 6a).

\section{Concluding remarks}

In Morales and Charbonneau $(2008,2009)$ we have proposed a new SOC model for solar flares, whose dynamical elements could easily be interpreted in terms of the physical picture of photospherically-forced coronal loops originally proposed 

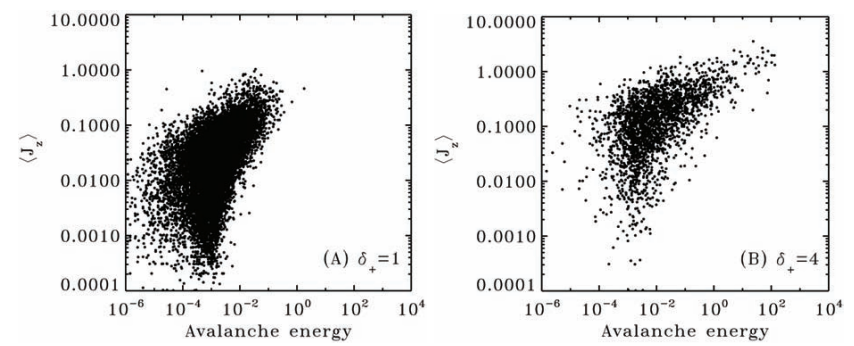

Fig. 6. Correlation relation between the avalanche energy and differential in mean electrical current $\left\langle J_{\mathrm{Z}}\right\rangle$ for two different values of the perturbation amplitude $\left(\delta_{+}\right)$. In panel (A) we show the result obtained for $\delta_{+}=1$ characterized by a Pearson correlation coefficient of 0.33 while in panel (B) we show the corresponding results for $\delta_{+}=4$ where correlation coefficient is 0.60 .

by Parker (1983) in the context of the coronal heating problem, but later shown to be applicable to flare energy release in general.

In the present paper we have modified the driving applied to such model and study the properties of the lattice energy, energy release and avalanche statistical properties. We observed that when increasing the amplitude of the positive driving the lattice energy increases slightly nevertheless the energy release by the lattice increases dramatically. We trace this behavior to the buildup of a net large-scale electrical current density flowing along the pseudo-coronal loop we identify with our lattice.

Because this large-scale current can act as an additional reservoir of energy, it is in retrospect perhaps not surprising that the probability distribution of flare size measures are affected by the presence of a systematic direction of twist in the simulation (cf. Fig. 4 herein). Interestingly, the steeper slopes in the probability distribution of total energy release are realized when forcing is symmetrical; this type of forcing is consistent with Parker's idea of random shuffling of the photospheric magnetic footpoints of coronal loops, producing a population of nanoflares; whereas the more strongly asymmetric forcing, yielding larger mean energy releases (cf. Fig. 3) because of the availability of an additional energy reservoir in the form of the mean loop-aligned current density, could be associated with higher energy flaring events, whose frequency distribution of energy release does show a power-law index of $\sim 1.5$, markedly smaller than the minimal value of 2.0 required by Parker's hypothesis of coronal heating by nanoflares. On the other hand, for the moderate assymmetrical forcing, the value of this index is very close to the observational results inferred by Uritsky et al. (2007). Ultimately, the distinction between symmetrical and asymmetrical forcing could hinge on the diameter of the loop, and where it is anchored in the solar photosphere.

\section{References}

Aschwanden, M. J.: Physics of the solar corona, Springer-Praxis Books in Astronomy and Planetary Sciences, Chichester, UK, 2006.

Aschwanden, M. J., Tarbell, T. D., Nightingale, R. W., Schrijver, C. J., Title, A., Kankelborg, C. C., Martens, P., and Warren, H. P.: Time Variability of the "Quiet" Sun Observed with TRACE. II. Physical Parameters, Temperature Evolution, and Energetics of Extreme-Ultraviolet Nanoflares, Astrophys. J., 535, 10471065, 2000.

Charbonneau, P., McIntosh, S. W., Liu, H.-L., and Bogdan, T. J.: Avalanche models for solar flares (Invited Review), Sol. Phys., 203, 321-353, 2001.

Dahlburg, R. B., Klimchuk, J. A., and Antiochos, S. K.: An Explanation for the "Switch-On" Nature of Magnetic Energy Release and Its Application to Coronal Heating, Astrophs. J., 622, 11911201, 2005.

De Moortel, I., Parnell, C. E., and Hood, A. W.: Determination of coronal loop properties from trace observations, Sol. Phys., 215, 69-86, 2003.

Georgoulis, M. K. and Vlahos, L.: Variability of the occurrence frequency of solar flares and the statistical flare, Astron. Astrophys., 336, 721-734, 1998.

Gomez, D.: Heating of the Solar Corona, Fundamentals of Cosmic Physics, 14(2-3), 131-233, 1990.

Isliker, H., Anastasiadis, A., and Vlahos, L.: MHD consistent cellular automata (CA) models. I. Basic features, Astron. Astrophys., 363, 1134-1144, 2000.

Klimchuk, J. A.: On Solving the Coronal Heating Problem, Sol. Phys., 234, 41-77, 2006.

Lopez Fuentes, M. C., Klimchuk, J. A., and Démoulin, P.: The Magnetic Structure of Coronal Loops Observed by TRACE, Astrophys. J., 639, 459-474, 2006.

Lu, E. T and Hamilton, R. J.: Avalanches and the distribution of solar flare, Astrophys. J., 380, L89-L92, 1991.

Mandrini, C. H., Demoulin, P., and Klimchuk, J. A.: Magnetic Field and Plasma Scaling Laws: Their Implications for Coronal Heating Models, Astrophys. J., 530, 999-1015, 2000.

Morales, L. F. and Charbonneau, P.: Self-organized Critical Model of Energy Release in an Idealized Coronal Loop, Astrophys. J., 682(1), 654-666, 2008.

Morales, L. F. and Charbonneau, P.: Geometrical Properties of Avalanches in a Pseudo-3D Coronal Loop, Astrophys. J., 698(2), 1893-1902, 2009.

Parker, E. N.: Magnetic Neutral Sheets in Evolving Fields - Part Two - Formation of the Solar Corona, Astrophys. J., 264, 642647, 1983.

Parker, E. N.: Nanoflares and the solar X-ray corona, Astrophys. J., 330, 474-479, 1988.

Podladchikova, O. and Lefebre, B.: Lattice models for solar flares and coronal heating (Invited Review), Solar Actvity and its Magnetic Origin, Proc. IAU Symposium, 233, 481-488, 2006.

Uritsky, V., Paczuki, M., Davila, J. M., and Jones, S. I.: Coexistence of Self-Organized Criticality and Intermittent Turbulence in the Solar Corona, Phys. Rev. Lett., 99(2), 25001-25001-4, 2007. 